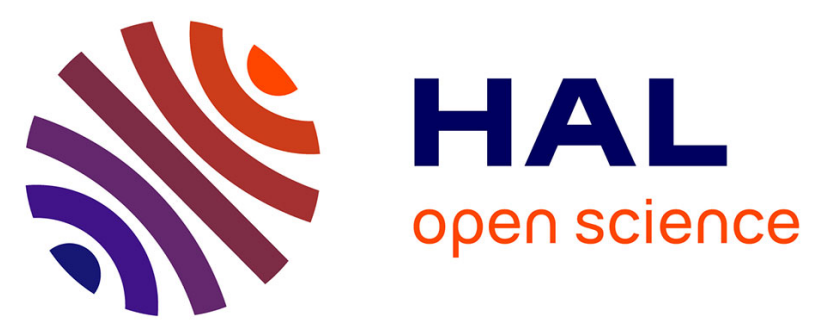

\title{
Nouvelle méthode de mesure rapide du pouvoir thermoélectrique aux très basses températures de petits échantillons. Application au cobalt monocristallin
}

A. Conan, G. Goureaux, M. Zoater

\section{- To cite this version:}

A. Conan, G. Goureaux, M. Zoater. Nouvelle méthode de mesure rapide du pouvoir thermoélectrique aux très basses températures de petits échantillons. Application au cobalt monocristallin. Revue de Physique Appliquée, 1971, 6 (3), pp.383-388. 10.1051/rphysap:0197100603038300 . jpa-00243560

HAL Id: jpa-00243560

https://hal.science/jpa-00243560

Submitted on 1 Jan 1971

HAL is a multi-disciplinary open access archive for the deposit and dissemination of scientific research documents, whether they are published or not. The documents may come from teaching and research institutions in France or abroad, or from public or private research centers.
L'archive ouverte pluridisciplinaire HAL, est destinée au dépôt et à la diffusion de documents scientifiques de niveau recherche, publiés ou non, émanant des établissements d'enseignement et de recherche français ou étrangers, des laboratoires publics ou privés. 


\title{
NOUVELLE MÉTHODE DE MESURE RAPIDE DU POUVOIR THERMOÉLECTRIQUE AUX TRÈS BASSES TEMPÉRATURES DE PETITS ÉCHANTILLONS. APPLICATION AU COBALT MONOCRISTALLIN
}

\author{
A. CONAN $(*)$, G. GOUREAUX et M. ZOATER \\ Laboratoire de Physique du métal et d'Electronique \\ Institut de Physique de l'Université de Nantes, B. P. 1044, Nantes
}

(Reçu le 2 avril 1971)

\begin{abstract}
Résumé. - Nous décrivons une nouvelle méthode de mesure du pouvoir thermoélectrique de petits échantillons poly ou monocristallins, dans la gamme de température 5-300 ${ }^{\circ} \mathrm{K}$. La méthode de mesure élimine les f. e. m. thermoélectriques parasites et est basée sur l'enregistrement des forces électromotrices thermoélectriques des couples plomb/échantillon et or- $0,03 \%$ fer/échantillon. Le principal avantage de la méthode est d'assurer un bon contact thermique et électrique des fils de mesure avec l'échantillon. Une application de cette méthode à la mesure du pouvoir thermoélectrique d'un monocristal de cobalt est donnée. Ces résultats sont comparés à ceux du magnésium dont le rapport $c / a$ est voisin de celui du cobalt.

Abstract. - A new technique is described for rapid measurement of the thermoelectric power of single crystal samples or brittle materials over a wide temperature range. The technique eliminates spurious thermal emf's in the leads and is based upon recording of the thermoelectric emf of the sample/lead-wire and the sample/Gold at $0,03 \%$ iron couples. The temperature gradient along the specimen is deduced. The main advantage of the method is that all the wires are in good thermal and electrical contact with the sample. As a test of this technique, the thermopower of a single crystal of cobalt was measured between 7 and $80^{\circ} \mathrm{K}$. A comparison with magnesium is given.
\end{abstract}

Introduction. - Il existe deux techniques de base pour la mesure des pouvoirs thermoélectriques [1]: la méthode différentielle et la méthode intégrale. Cette dernière nécessite l'utilisation d'échantillons d'assez grande longueur et consiste à mesurer la f. e. m. thermoélectrique $V(T)$ d'un couple dont la soudure froide est maintenue à température constante $\left(4,2{ }^{\circ} \mathrm{K}\right.$ par exemple), l'autre soudure est chauffée à la température $T$. La dérivée de la courbe $V(T)$ par rapport à la température permet d'accéder au pouvoir thermoélectrique par la relation

$$
Q=\frac{\mathrm{d} V(T)}{\mathrm{d} T} .
$$

Cette méthode n'est en général pas applicable aux échantillons monocristallins qui se présentent en général sous la forme de cubes ou cylindres de fortes sections et de faible longueur. La méthode différentielle consiste alors à appliquer un faible gradient de température $\Delta \mathbf{T}$ à l'échantillon et à mesurer l'accroissement $\Delta(E)$ de la f. e. $\mathrm{m}$. thermoélectrique correspondante. Le pouvoir thermoélectrique de l'échantillon est alors donné par :

$$
Q=\frac{\Delta E}{\Delta T}
$$

(*) Travail effectué dans le cadre de la thèse de doctorat ès Sciences Phys. de M. A. Conan (enregistrée au C.N.R.S. sous le $n^{0}$ AO 5437).
Pour que cette relation soit valable, il faut que le gradient de température le long de l'échantillon soit faible, que l'échantillon soit homogène et que le champ électrique créé le long de l'échantillon ait même direction que le gradient de température. Les premières difficultés que l'on rencontre lors de la mesure du pouvoir thermoélectrique sont le maintien à une température uniforme, l'élimination des f. e. m. parasites et l'entretien d'un gradient de température constant. L'utilisation d'appareils adaptés (régulation de température, source de tension continue en opposition) permet de surmonter les deux premières difficultés.

Rowe [2] a montré que le dernier problème pouvait être résolu en soumettant l'échantillon à de très faibles échelons de température $\left(\Delta T_{i}\right)$ et en mesurant les variations de f.e. $m$. thermoélectrique correspondantes $\left(\Delta V_{i}\right)$. La courbe $\Delta V_{i}=\mathrm{f}\left(\Delta T_{i}\right)$ est alors linéaire et sa pente donne le pouvoir thermoélectrique de l'échantillon. Cependant ce procédé de mesure est très long car il est nécessaire d'attendre un état d'équilibre du système après chaque accroissement de température. Un autre procédé de mesure utilisé par Caskey, Sellmyer et Rubin [3] est basé sur la mesure simultanée de $\Delta T$ et $\Delta V$ lorsque le gradient de température évolue lentement. Nous avons amélioré cette dernière méthode.

Méthode de mesure. - Lorsque le gradient de température varie le long de l'échantillon, il est en général très difficile de le mesurer avec précision. Deux 
méthodes sont alors possibles : - mesurer la température des deux faces de l'échantillon à l'aide de sondes ou de thermocouples. Cette méthode n'est pas satisfaisante car elle conduit à mesurer des f. e. m. de l'ordre du millivolt et à les retrancher pour retrouver une valeur de l'ordre du microvolt ; - utiliser un thermocouple monté en différentiel (Fig. 1a). Cette dernière méthode a le désavantage que l'une des extrémités du thermocouple utilisé ne peut pas être en contact direct avec l'échantillon sans court-circuiter ce dernier. Or, étant données leurs faibles épaisseurs, cette erreur peut devenir très importante en valeur relative.

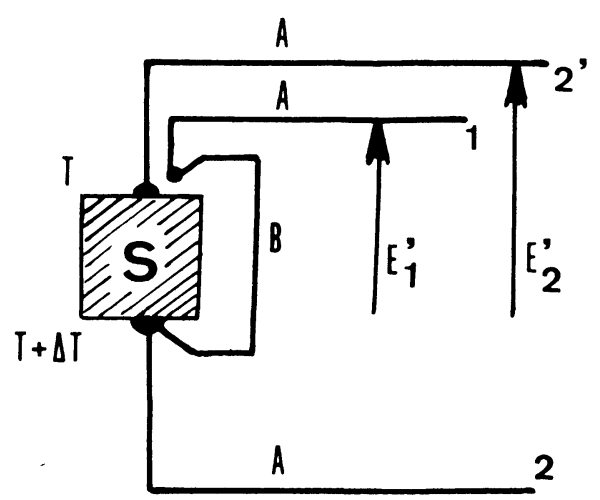

FIG. $1 a$.

Nous-avons imaginé une troisième méthode qui permet d'éviter le problème du contact thermique avec l'échantillon. Considérons le circuit de la figure $1 b$ et désignons par S l'échantillon soumis au faible gradient

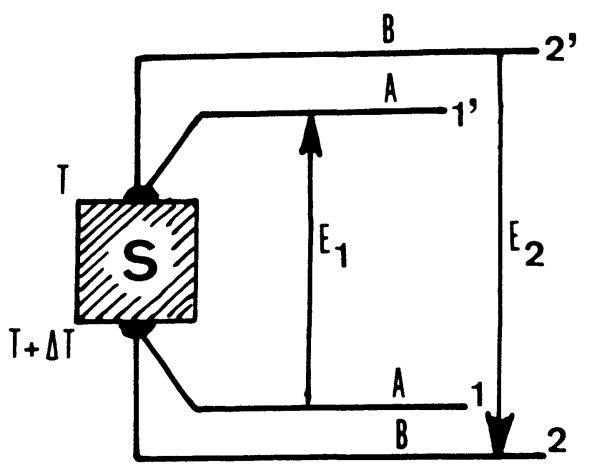

FIG. $1 b$.

de température $\Delta T$. Les fils A et $\mathrm{B}$ sont en contact thermique et électrique direct avec l'échantillon. Sur le circuit thermoélectrique constitué par l'échantillon et le métal $A$ on mesure entre les bornes 1 et $1^{\prime}$ une f. e. $m$ de Seebeck :

$$
E_{1}=\int_{T}^{T+\Delta T} \mu_{\mathrm{S} / \mathrm{A}} \mathrm{d} T+\pi_{\mathrm{S} / \mathrm{A}}(T+\Delta T)-\pi_{\mathrm{S} / \mathrm{A}}(T)
$$

où $\mu_{\mathrm{SA}}$ et $\pi_{\mathrm{SA}}$ sont respectivement les coefficients d'effet Thomson et Peltier de l'échantillon par rapport au métal A. De même que le circuit constitué par l'échantillon et le métal $\mathrm{B}$ apparaît entre les bornes 2 ' et 2 la f. e. m. thermoélectrique :

$$
E_{2}=\int_{T}^{T+\Delta T} \mu_{\mathrm{B} / \mathrm{S}} \mathrm{d} T+\pi_{\mathrm{B} / \mathrm{S}}(T+\Delta T)-\pi_{\mathrm{B} / \mathrm{S}}(T) .
$$

On obtient donc par addition de ces deux équations :

$$
\begin{aligned}
E_{1}+E_{2}=\int_{T}^{T+\Delta T} \mu_{\mathrm{B} / \mathrm{A}} \mathrm{d} & + \\
& +\pi_{\mathrm{B} / \mathrm{A}}(T+\Delta T)-\pi_{\mathrm{B} / \mathrm{A}}(T) .
\end{aligned}
$$

Cette expression n'est autre que la f. e. m. de Seebeck du métal B par rapport au métal $\mathrm{A}$. Il suffit donc de choisir deux métaux A et B qui constituent un thermocouple étalonné pour accéder à l'expression du pouvoir thermoélectrique de l'échantillon par rapport au métal A :

$$
\begin{gathered}
Q_{\mathrm{S} / \mathrm{A}}=\frac{E_{1}}{E_{1}+E_{2}}\left[\frac{\mathrm{d}\left(E_{1}+E_{2}\right)}{\mathrm{d} T}\right]_{\bar{T}}=\frac{E_{1}}{E_{1}+E_{2}} Q_{\mathrm{B} / \mathrm{A}}(\bar{T}) \\
\bar{T} \simeq T+\frac{\Delta T}{2} .
\end{gathered}
$$

Or le pouvoir thermoélectrique $Q_{\mathrm{B} / \mathrm{A}}(\bar{T})$ peut être déterminé de façon très précise par une méthode intégrale.

On peut donner un ordre de grandeur de l'erreur commise sur le rapport (*)

$$
\begin{gathered}
R=\frac{E_{1}}{E_{1}+E_{2}} \\
\left|\frac{\Delta R}{R}\right|=\frac{\Delta E_{1}}{E_{1}}-\frac{\Delta\left(E_{1}+E_{2}\right)}{E_{1}+E_{2}} \\
=\frac{1}{E_{1}+E_{2}}\left[\left|\frac{E_{2}}{E_{1}}\right|\left|\Delta E_{1}\right|+\left|\Delta E_{2}\right|\right] .
\end{gathered}
$$

Si on admet que les erreurs absolues sur les mesures de $E_{1}$ et $E_{2}$ sont égales, il vient :

$$
\left|\frac{\Delta R}{R}\right| \simeq \frac{\Delta E_{1}}{E_{1}}
$$

Ce procédé de mesure a non seulement l'avantage d'assurer un excellent contact thermique des couples avec l'échantillon mais encore il permet d'obtenir une précision deux fois meilleure que le procédé décrit figure $1 a$.

Description de la cellule de mesure. - La cellule de mesure (Fig. 2) est fixée sur la queue d'un cryostat à température variable T. B. T. Sa partie supérieure est un bloc de cuivre cylindrique percé d'un trou oblique de $1 \mathrm{~mm}$ de diamètre destiné au passage des fils de

(*) Remarque : La quantité $\left(E_{1}+E_{2}\right)$ qui intervient au dénominateur de $R$ représente l'accroissement de f.e. m. thermoélectrique du couple étalonné dans la gamme de température envisagée. Cette quantité n'est donc nulle que si $E_{1}$ et $E_{2}$ sont nuls, c'est-à-dire pour $\Delta T=0$. 
thermocouples. Ce bloc a été conçu pour recevoir deux sondes : la première (4) sert à la régulation de la température de l'extrémité de la cellule. La seconde sert à la mesure de la température de l'échantillon. C'est une diode TG $100 \mathrm{~K}$ de chez Philartron, de sensibilité égale à $1,5 \mathrm{mV} /{ }^{\circ} \mathrm{K}$ à $4,2^{\circ} \mathrm{K}$. Elle est étalonnée à $\pm 0,01{ }^{\circ} \mathrm{K}$ de 4,2 à $10^{\circ} \mathrm{K}$ et à $\pm 0,05^{\circ} \mathrm{K}$ de 10 à $70^{\circ} \mathrm{K}$. Ces deux diodes sont isolées électriquement par un dépôt sur leurs parties actives d'un vernis à grande

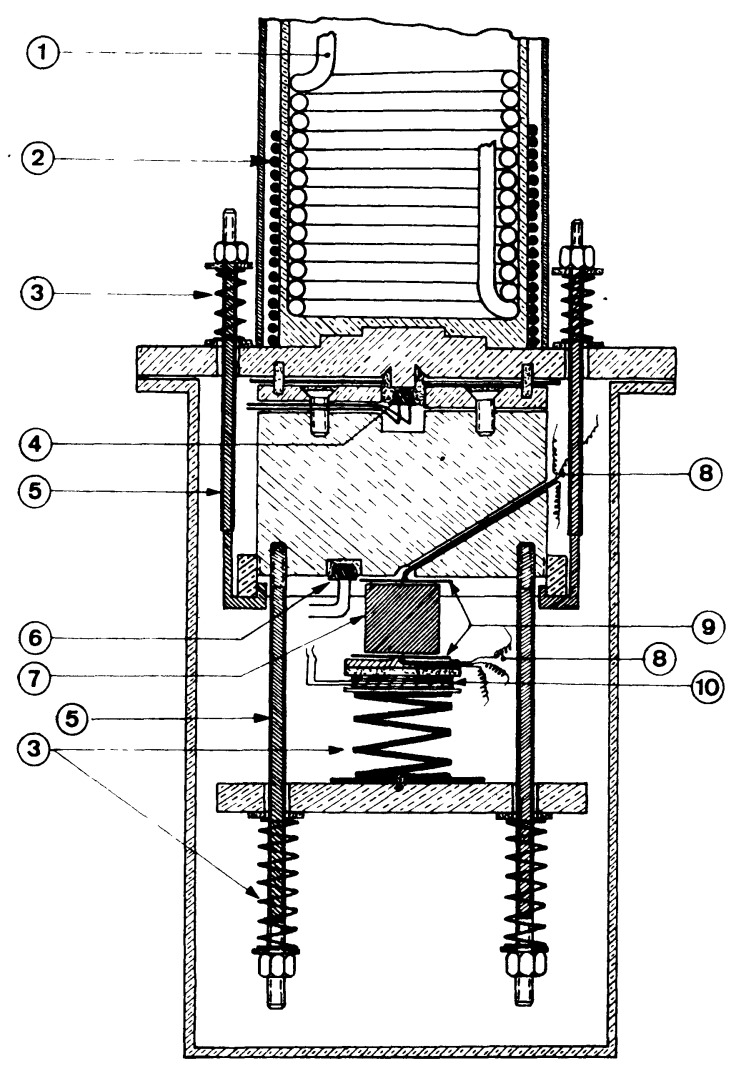

FIg. 2. - (1) Serpentins (vapeurs d'Hélium); (2) Four principal ; (3) Ressorts (bronze-beryllium) ; (4) Sonde de régulation de température; (5) Tirants gainés de téflon; (6) Sonde de mesure de la température de l'échantillon; (7) Echantillon; (8) Thermocouples $(\mathrm{Au}-0,03 \% \mathrm{Fe}, \mathrm{Pb}$ et $\mathrm{Ni})$; (9) Clinquants d'or ; (10) Four secondaire. conductibilité thermique de chez General Electric. Leur contact thermique est assuré par de la poudre de cuivre mélangée à de la graisse conductrice.

Les fils de thermocouples (8) sont fixés à l'aide d'une soudure spéciale libre de force électromotrice thermoélectrique Keitley sur une feuille d'or (9) d'épaisseur 1 micron. Elle assure un meilleur contact thermique et électrique avec l'échantillon. Enfin, des volants thermiques constitués par des enroulements de ces fils sur des barreaux de téflon permettent de minimiser les fuites thermiques.

La partie inférieure de la cellule est réalisée de la même façon que la précédente. Elle est munie en son extrémité inférieure d'un petit four plat (10) destiné à contrôler le gradient de température auquel est soumis l'échantillon. Il est donc nécessaire que cette pièce présente l'inertie thermique la plus faible possible, aussi ses dimensions sont-elles très inférieures à celles du bloc supérieur.

L'échantillon (7) est placé entre les deux blocs et maintenu en contact avec la partie supérieure par l'intermédiaire de ressort en alliage à base d'oxyde de Béryllium (3). Tous les fils de connection sont légèrement torsadés et soudés à des fils de cuivre pur. Ces soudures sont placées en contact thermique direct sur le réservoir azote du cryostat. A la sortie du cryostat ces fils de cuivre pur sont raccordés à des fils blindés de même nature.

Choix des thermocouples. Circuit de mesure. Pour obtenir une bonne précision de mesure, il est nécessaire de disposer d'un thermocouple à fort pouvoir thermoélectrique dans le domaine de température envisagé. Or le couple " alliage or (spectroscopiquement pur) $+0,03 \%$ Fer-Chromel » [4] [5] qui possède un pouvoir thermoélectrique (Fig. $3 a$ ) compris entre 10 et $15 \mu \mathrm{V}$ dans le domaihe exploré, semble répondre à nos exigences, de préférence à un couple alliage or $+\%$ cobalt dont la reproductibilité est moins bonne. Comme le P. T.E. absolu d'un métal est une grandeur physique caractéristique de ce métal, il est donc nécessaire de retrancher de nos résultats le P. T.E. absolu de

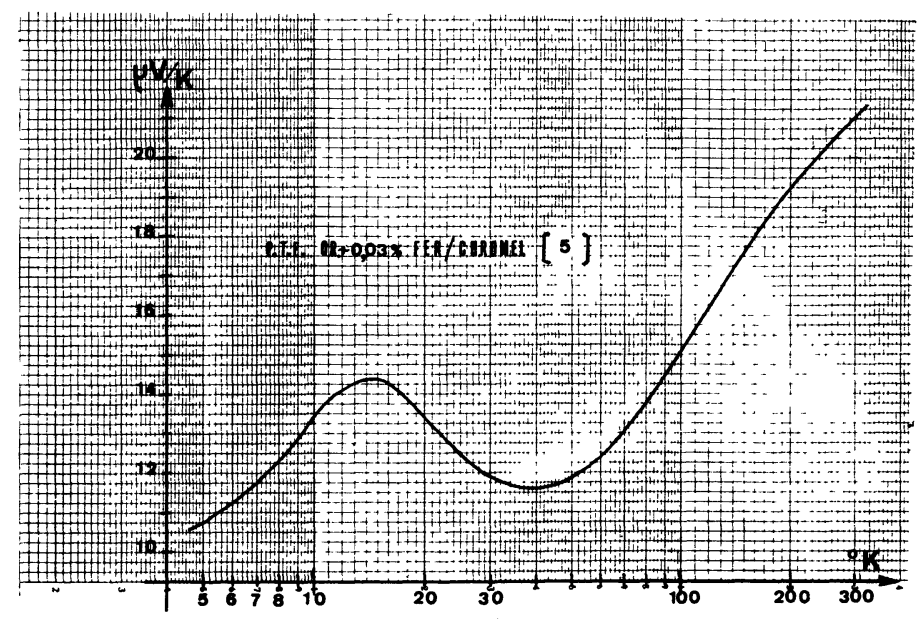

FIG. $3 a$. 
l'alliage (Au-0,03\% Fe) (Fig. 3b) ; ceci pouvait entraîner une erreur importante dans nos mesures car nous ne disposions pas d'un fil (Au-Fe) parfaitement étalonné. Nous avons donc préféré associer ce métal au plomb qui définit en quelque sorte l'échelle thermoélectrique absolue. Le pouvoir thermoélectrique de ce couple est reporté (Fig. $3 c$ ) et on peut constater que son $P$. T. E. reste appréciable dans la gamme de température $4,2-80 \circ \mathrm{K}$ mais devient trop faible pour des températures plus élevées.

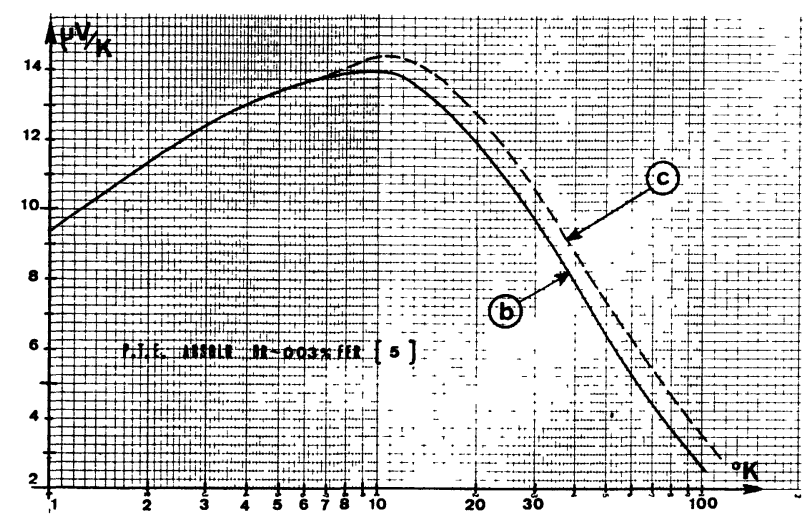

FIG. $3 b$ et $c$.

Pour vérifier nos résultats dans la gamme 80$300^{\circ} \mathrm{K}$ nous avons recherché un métal qui, associé au plomb, donnerait un P. T. E. élevé et permettrait un recoupement de nos résultats avec le couple $(\mathrm{Au} \% \mathrm{Fe}$ $\mathrm{Pb}$ ) au voisinage de $50^{\circ} \mathrm{K}$. Le Nickel étudié par de nombreux auteurs [7], [8], [9], [10] possède un pouvoir thermoélectrique absolu de $-7 \mu \mathrm{V} /{ }^{\circ} \mathrm{K}$ à $50^{\circ} \mathrm{K}$ et de $-17 \mu \mathrm{V} /{ }^{\circ} \mathrm{K}$ à $250^{\circ} \mathrm{K}$ et donne la solution de ce problème. Ceci nous a amené à réaliser des soudures triples $\mathrm{Au}+\% \mathrm{Fe}, \mathrm{Pb}, \mathrm{Ni}$. De nombreuses précautions doivent être prises pour minimiser les forces électromotrices parasites : la soudure $\mathrm{Au}+\% \mathrm{Fe}-\mathrm{Ni}$ est faite sans apport de métal intermédiaire en opérant par « claquage » dans de l'huile de parafine pour prévenir toute oxydation. Comme cette soudure est à haut point de fusion, nous pouvons ensuite y fixer le fil de plomb à l'aide de la soudure libre de f. e. m. thermoélectrique "Keitley » à bas point de fusion.

Le schéma électrique de mesure est reporté (Fig. 4). A la diode de mesure de température (6) se raccordent quatre fils de cuivre émaillé. Les deux premiers servent à l'alimenter à courant constant $(100 \mu \mathrm{A})$, les deux autres à mesurer la f. e. m. qui apparaît à ses bornes. Le montage à quatre fils n'est pas nécessaire pour la sonde de régulation. Les fils (8) des divers couples sont raccordés à des fils de cuivre (11) et toutes les soudures sont ramenées au même point de référence $T_{0}$. Ce dernier a été obtenu en pratiquant un puits dans l'écran d'azote du cryostat dans lequel sont plongées les soudures. Le contact thermique est assuré par de la poudre de cuivre mélangée à un peu de graisse à vide. L'orifice est obturé par une feuille de clinquant d'or

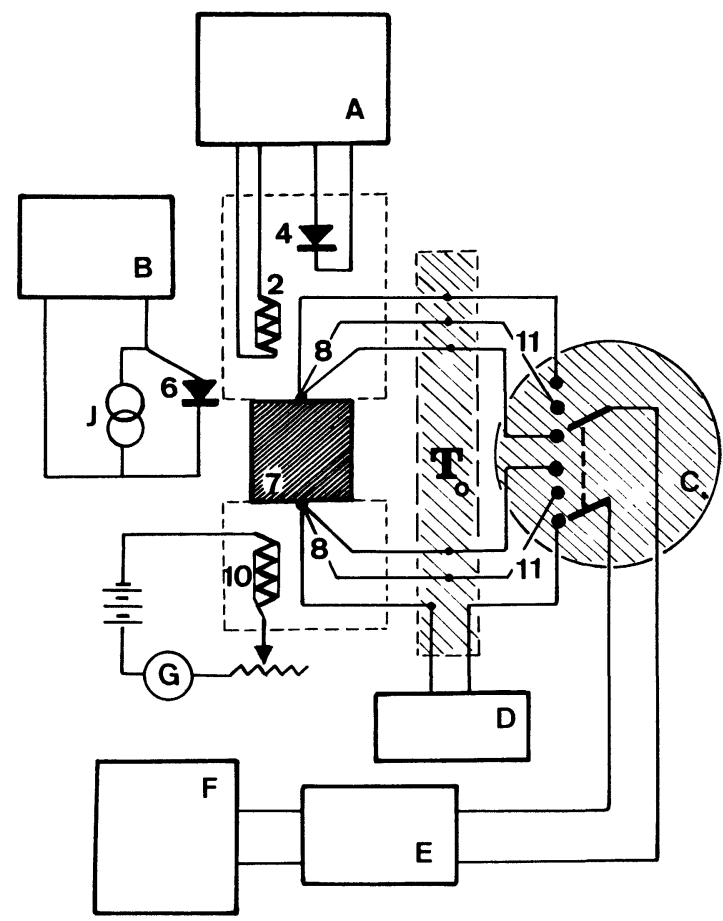

Fig. 4. - (A) Régulation de température Par 152 ; (B) Microvoltmètre digital Solartron; (C) Commutateur thermostaté Tinsley (4092-D); (D) Source de nanovolt Keitley 460; (E) Nanovoltmètre Keitley 148 ; (F) Enregistreur Meci speedomax; (G) Milliampèremètre; (J) Source de courant stabilisée ; (2) Chauffage principal ; (4) Sonde de régulation ; (6) Sonde de mesure de la température de l'échantillon; (7) Echantillon; (8) Couples $(\mathrm{Au}-0,03 \% \mathrm{Fe}, \mathrm{Pb}$ et $\mathrm{Ni})$; (10) Four secondaire;

(11) Fils de cuivre de sortie des couples du cryostat.

pour éviter les pertes par rayonnement. Remarquons que $T_{0}$ n'est pas une "référence " au sens propre du terme puisqu'en réalité il nous suffit que deux fils de même nature restent à la même température. C'est le pouvoir thermoélectrique élevé du couple NickelCuivre qui nous a amené à prendre cette précaution supplémentaire. La sortie du cryostat se fait par l'intermédiaire de passages étanches munis de fils de cuivre de même nature scellés à l'araldite. Les bornes communes du commutateur $\mathrm{C}$ 《Tinsley » à quatre positions (la quatrième est une position de courtcircuit et sert uniquement à faire le zéro des appareils de mesure) sont reliées au navovoltmètre $\mathrm{E}$ utilisé en amplificateur. Cette utilisation est rendue possible grâce au fait qu'ayant à mesurer le quotient de deux grandeurs cette valeur est indépendante du gain de l'amplificateur. La sortie de E est reliée par l'intermédiaire d'un atténuateur à un enregistreur F. L'atténuateur sert uniquement à l'adaptation de $\mathrm{F}$ à $\mathrm{E}$.

Exploitation des résultats. - Un diagramme relatif à une mesure est reporté figure 5 et illustre particulièrement bien la méthode de mesure. Il se décompose en trois parties qui correspondent aux différentes phases de la mesure.

- La partie 1 du diagramme est relative à la période de chauffage maximum du four principal. Son 
origine est telle que le gradient de température est nul dans l'échantillon à la température $T_{1}$. La nouvelle température $T_{2}$ affichée sur le potentiomètre de la régulation est supérieure de $4{ }^{\circ} \mathrm{K}$ environ à la température précédente, et est atteinte en moins de deux minutes. Cette zone montre une croissance rapide des f. e. $\mathrm{m}$. thermoélectriques des couples plomb-échantillon et nickel-échantillon. Puis la température de la face supérieure de l'échantillon se stabilise à $T_{2}$ et reste constante durant la suite des mesures.

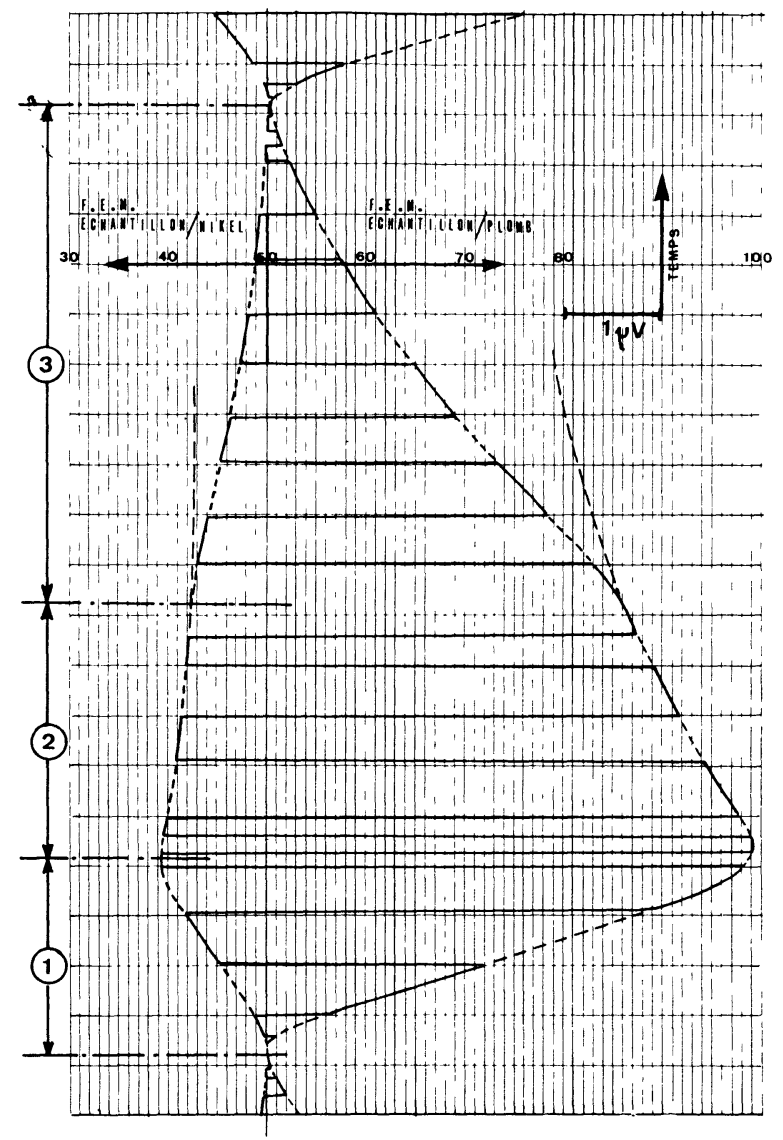

FIG. 5.

- La partie 2 du diagramme montre que les courbes passent par des maxima et commencent à décroître exponentiellement. Cette zone correspond à une diminution du gradient de température due à la conduction de chaleur le long de l'échantillon.

- Après une attente de deux minutes environ nous accélérons cette descente (partie 3 du diagramme) par chauffage de la partie inférieure de l'échantillon. L'alimentation du four auxiliaire (10) est coupée un peu avant l'intersection des deux courbes et immédiatement après nous affichons un nouveau point qui correspond à une température plus élevée pour recommencer un nouveau cycle.

Il est facile de constater sur ce diagramme que les couples n'ont pas de retard l'un par rapport à l'autre puisque la position des maxima coïncide, et que la dérive due au f. e. m. parasites est faible puisque les courbes se recoupent pratiquement sur la droite origine. L'enregistrement de la totalité du diagramme permet d'obtenir un bon recoupement pour nos mesures : pour chaque point affiché nous notons le rapport des maxima et le comparons à la pente de la droite obtenue en reportant sur un même graphique les points expérimentaux $E_{\mathrm{S} / \mathrm{Ni}}$ en fonction de $E_{\mathrm{S} / \mathrm{Pb}}$. Le rapport des maxima doit toujours être compris entre les deux valeurs des rapports de pentes qui l'encadrent.

Remarque. - Les premières mesures que nous effectuons sont en général un peu moins précises que les suivantes. En effet, lors du remplissage du cryostat en Hélium liquide il existe un assez fort gradient dans l'échantillon. Ce gradient diminue dès la fin du remplissage mais la différence de température entre les deux faces de l'échantillon est encore de l'ordre de $2{ }^{\circ} \mathrm{K}$ lorsque nous commençons à manipuler. Pour la première mesure, nous faisons un "zéro » artificiel sur nos circuits à l'aide d'une source de nanovolts placée en opposition sur l'un des circuits de mesure puis nous chauffons pendant un court instant avec le four bas. Les deux mesures suivantes sont faites de façon à amener un gradient nul dans l'échantillon et ce, par action prolongée de l'un ou l'autre des fours. La correction introduite est calculée pour ces deux mesures lors $\mathrm{du}$ dépouillement des résultats.

Résultats relatifs au cobalt monocristallin. - Les résultats expérimentaux portant sur la mesure du pouvoir thermoélectrique du Cobalt sont peu nombreux. Les travaux les plus récents ont été effectués sur du Cobalt polycristallin dans les domaines des hautes températures $(300$ à 1600 \% $)$ [11] et des très basses températures $\left(1,2-6^{\circ} \mathrm{K}\right)$ [12].

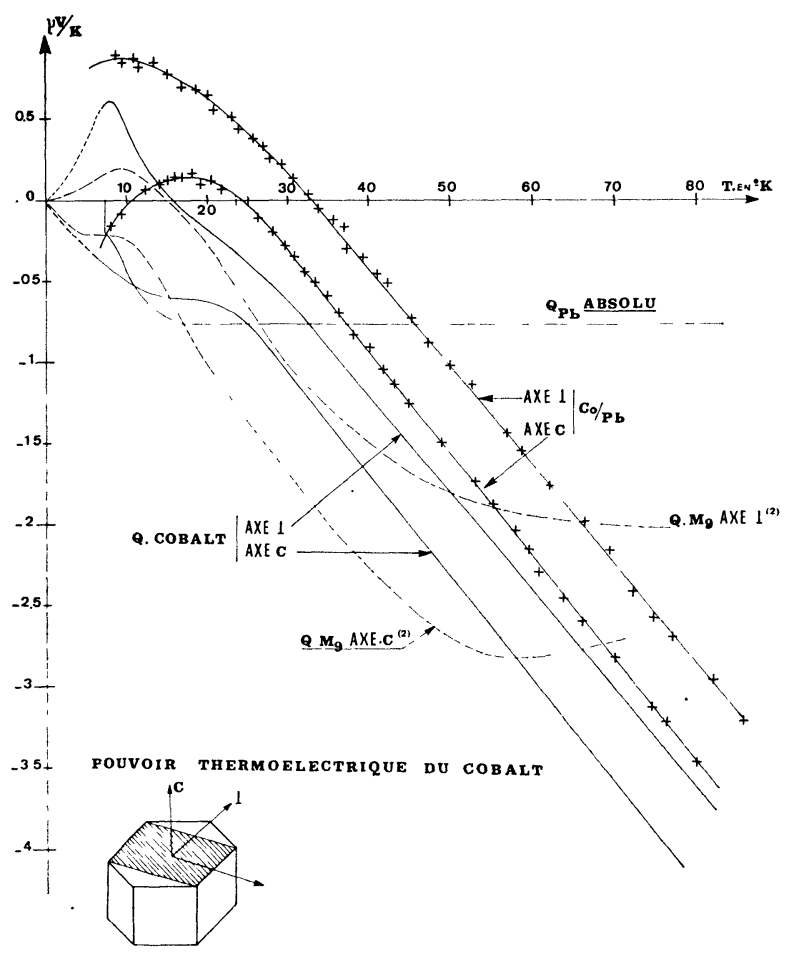

FIG. 6. 
Dans une structure hexagonale le tenseur thermoélectrique a deux coefficients indépendants $Q_{\|}$et $Q_{\perp}$ qui correspondent à des mesures faites parallèlement et perpendiculairement à l'axe c du cristal. Notre échantillon se présente sous la forme d'un petit cube de $9 \mathrm{~mm}$ de côté environ dont les faces sont parallèles à l'arc près et est taillé selon le schéma de la figure 6. Sa pureté est de $99,98 \%$ environ. Nous avons reporté sur un même graphique (Fig. 6) les résultats de nos mesures du pouvoir thermoélectrique du cobalt par rapport au plomb selon ses axes perpendiculaire et parallèle à l'axe $c$, les courbes - déduites de ces dernières - du pouvoir thermoélectrique absolu du cobalt et les résultats de Rowe [2] relatifs au P. T. E. du magnésium. La composante perpendiculaire du pouvoir thermoélectrique absolu du cobalt est positive à très basse température, passe par un maximum au voisinage de $10^{\circ} \mathrm{K}$ puis décroît pour s'annuler à $15 \mathrm{~K}$. Au-delà de $30^{\circ} \mathrm{K}$ la variation du pouvoir thermoélectrique est pratiquement linéaire. L'axe $c$ présente toujours un pouvoir thermoélectrique négatif avec une légère anomalie dans la courbure entre 10 et $20^{\circ} \mathrm{K}$.

Conclusions. - A. Le dispositif expérimental adopté permet d'obtenir une bonne reproductibilité des mesures et présente de nombreux avantages :

- Détermination précise du gradient de température qui existe entre les deux points d'arrivée des fils de mesure.

- Possibilité de travailler au voisinage d'un gradient de température nul.

- Elimination des f. e. m. parasites en enregistrant des variations de f. e. $m$. thermoélectriques et ne s'intéressant qu'aux pentes des droites obtenues.
- Rapidité et précision de mesures très nettement améliorées par rapport aux dispositifs couramment utilisés.

B. Il est d'autre part bien connu que le pouvoir thermoélectrique d'un métal est étroitement lié à la forme de sa surface de Fermi. La surface de Fermi du Cobalt a récemment été calculée théoriquement [13] par la méthode de fonction de Green (K. K. R.). Ces résultats peuvent être comparés à ceux obtenus pour le magnésium par la méthode O. P. W. [14], [15], [16]. Le magnésium a en effet un rapport $\frac{c}{a}$ (axe $c /$ axe perpendiculaire) égal à 1,6222 qui est très voisin de la valeur 1,6322 du cobalt. Il n'est donc pas étonnant que les courbes du pouvoir thermoélectrique du cobalt et $d u$ magnésium (Fig. 6) présentent de grandes analogies. Nos résultats confirment donc les conclusions générales de Rowe [2].

A basse température, le signe du pouvoir thermoélectrique est déterminé par la nature (électron ou trou) de la partie de la surface de Fermi (monstres-capslentilles-papillons-aiguilles ou cigares) de plus petite dimension.

Lorsque la température croît les contributions des autres régions de la surface de Fermi prédominent successivement et dans le même ordre de grandeur que la croissance de leur dimension.

A haute température, lorsque plusieurs parties de la surface de Fermi contribuent au dragage phonique les corrélations entre pouvoir thermoélectrique et surface de Fermi sont plus confuses et le problème est rendu encore plus compliqué par la présence d'interactions phonons-phonons de type $U$.

\section{Bibliographie}

[1] White (G. K.), Experimental techniques in low temperature, Clarendon Press, $1^{\text {re }}$ éd., 1959.

[2] Rowe (V. A.), Schroeder (P. A.), J. Phys. Chem. Sol., 1970, 31, 1.

[3] Caskey (G. R.), Sellmyer (D. J.), Rubin (L. G.), Rev. Sci. Instr., 1969, 40, 1280.

[4] Berman (A.), Brock (V. C. F.), Huntley (D. J.), Cryogenics, 1964, 4, 233

[5] Rosembaum (R. L.), Rev. Sc. Instr., 1968, 39, 6890.

[6] Thoulouze (D.-J.), Phys. France supp. au no 10, 1970, C 3, 119.

[7] Vedernikov (M. V.), adv. in Phys., G. B., 1969, 18, $174,337$.

[8] Christian Jan Pearson Templeton, Proc. Roy. Soc. 1958, A 245, 217.
[9] Grieg (D.), Harisson (J. P.), Phil. Mag., 1965, $12,71$. [10] Blatt (F. J.), Flood (D. J.), Rowe (V.), Schroeder (P. A.), Cox (J. E.), Phys. Rev. Letters, 1967, 18, 395.

[11] Schroeder (K.), Giannuzzia, Phys. Stat. Solid, 1969, 34, 133.

[12] Radhakrishna Nielsen (M (P.), Phys. Stat. Solid, 1965, 11, 111.

[13] Wakoh (S.), Yamashita (J.), J. Phys. Soc., 1970, 28, 1151.

[14] Falicov (L. M.), Phil. Trans. Roy. Soc., 1963, A 255, 55.

[15] Ketterson (J. B.), Stark (R. W.), Phys. Rev., 1967, 156, 748 .

[16] Priestly (M. E.), Proc. Roy. Soc., 1963, A 276, 258. 